\title{
Pediatric testicular microlithiasis through four clinical case studies: review of the literature and proposal of clinical guidelines
}

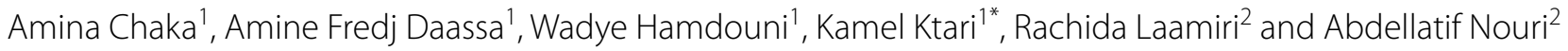

\begin{abstract}
Background: Testicular microlithiasis in children was defined for the first time in 1961 based on histological criteria. There should be more than 5 calcifications per testicle in order to say that the patient has testicular microlithiasis. It has three different echographic grades depending on the number of calcifications. However, this disease is uncommon, with inaccurate prevalence and no certain information about its evolution or etiology.
\end{abstract}

Main body: We studied 4 clinical cases of children diagnosed with testicular microlithiasis, in light of the conducted review of the literature, and we defined the characteristics of this disease and proposed a management and monitoring framework based on the clinical observations.

Conclusion: There is a link between testicular microlithiasis and testicular cancer. Therefore, it is recommended to make a regular follow-up of children who present testicular microlithiasis with the presence of risk factors.

Keywords: Testicular microlithiasis, Calcifications, Echographic grade, Framework, Scrotal ultrasound

\section{Background}

Based on ultrasound criteria, testicular microcalcifications are defined as multiple small hyper-echoic signals, usually without posterior acoustic shadowing, spreading diffusely in the testicular parenchyma. Their size is small, mainly between 1 and $3 \mathrm{mms}$ [1], and is either unilateral or bilateral. Ultrasound should count more than 5 calcifications to define microlithiasis and determine its echographic grade. In fact, there are three grades defined as follows: grade $1-5$ to $10 \mathrm{MT}$ per field; grade 2-from 10 to 20; and grade 3-with more than 20 TM per field $[1,2]$.

This disease is relatively uncommon [1] and raises the following problems: unknown etiology, inaccurate

\footnotetext{
*Correspondence: ktari-kamel@hotmail.fr

${ }^{1}$ Department of Urology, Fattouma Bourguiba Hopital, Monastir, Tunisia Full list of author information is available at the end of the article
}

prevalence, unpredictable long-term evolution, possibly leading to cancer or infertility, and finally lack of standardized management and monitoring procedures.

Hence, the objective of the present study is to understand this disease and suggest a management framework based on observations and literature data.

\section{Main body}

In order to study this disease, we used medical records of 4 children diagnosed with TMs between 2003 and 2016 in the Pediatric Surgery Department. To confirm the diagnosis, we used both ultrasound and histological examination. The methodology of our work began with data collection, in which we used different techniques: epidemiological, clinical, biological, radiological, pathological, and different therapeutic modalities as well as a follow-up of the above children cases in 
comparison with those discussed in the medical literature about this disease.

\subsection{Observation 1}

The first case concerns an 11-year-old child, with no important medical history, referred to our Emergency Department for testicular trauma.

The physical examination was normal, after which we concluded that both testicles were painless and properly placed, with normal consistency, yet appeared to be bilaterally inflamed.

A scrotal ultrasound was performed, and the resulting images showed an hematocele on the right side and many scattered bilateral microlithiases of grade 3 (Fig. 1).

In order to predict the development of any tumor because of radiology finding, we ordered a testicular ultrasound to be done two weeks later and an Alpha FP rate, since it could recur as follows: First, hematocele will disappear, while the TMs will develop and persist and the Alpha FP rate will be normal.

\subsection{Observation 2}

The second case concerns a 1- and a half-year-old child, referred for the left testicular ectopia neonatal diagnosis. We did first a general examination and did not identify any abnormalities. The examination of the testicles showed that the left one was an ectopic inguinal testicle, while the right one was properly placed with abnormal size and consistency.

Given the ultrasound image, it is observed that the left testicle is $18 \times 8 \times 10 \mathrm{~mm}$ hyper-echoic, homogeneous and inguino-scrotal. Moreover, the right testicle is $15 \times 10 \times 10 \mathrm{~mm}$ hyper-echoic with delicate intra-scattered glandular calcifications (Figs. 2, 3).

Since these ultrasound images showed a suspicious appearance, we decided to have an AFP and bHCG tumor marker dosage, which were found normal.

The pathological result of the biopsy of both testicles showed the presence of more bilateral TMs on the left side. The discovered TMs were not detected on the ultrasound previously done.

As a follow-up, our patient undertook tumor markers' tests and testicular ultrasound every 6 months. After 2 years of follow-up tests, we observed
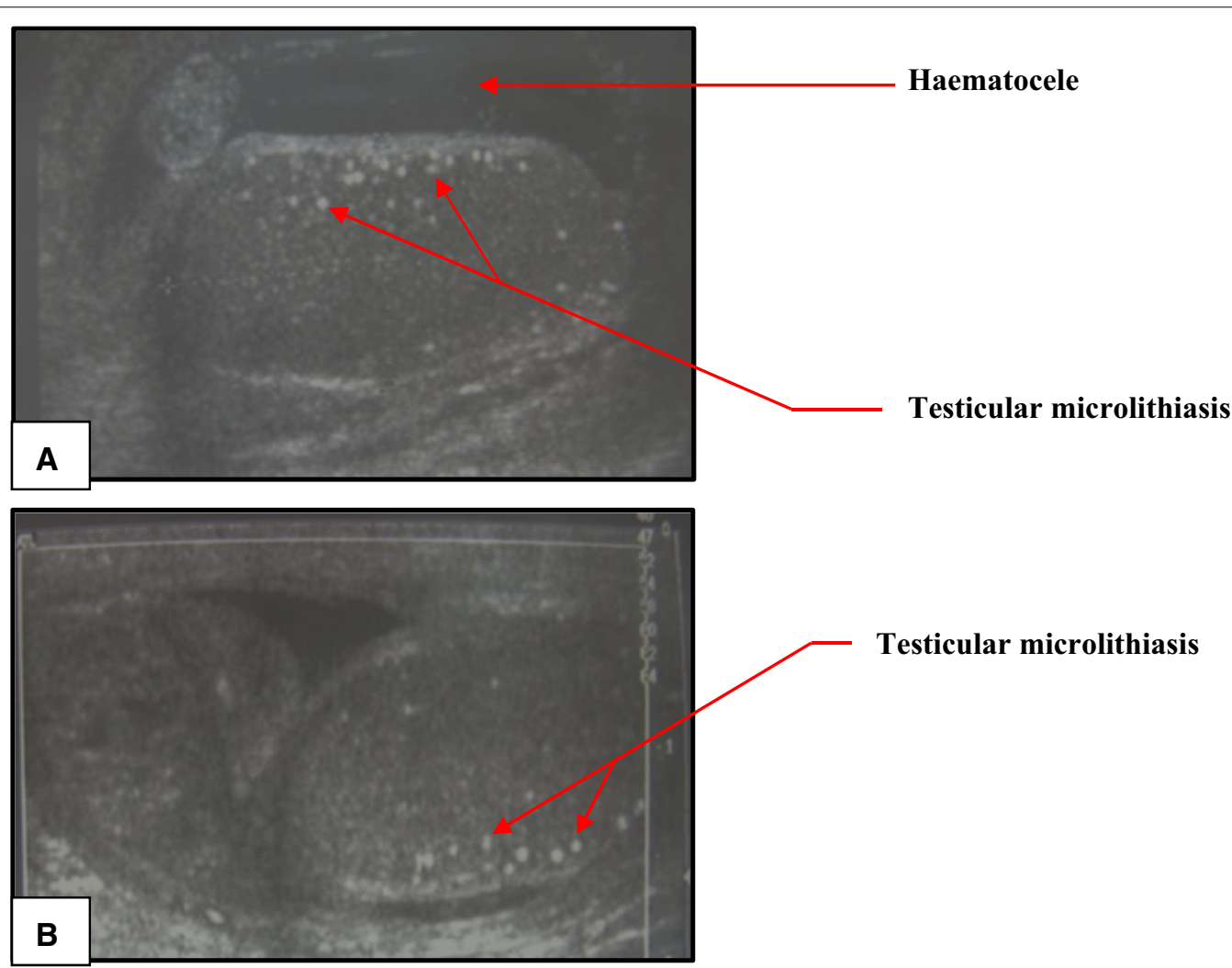

Fig. $1 \mathbf{A}$ and $\mathbf{B}$ Echographic image showing bilateral TMs and hematocele on the right testis 


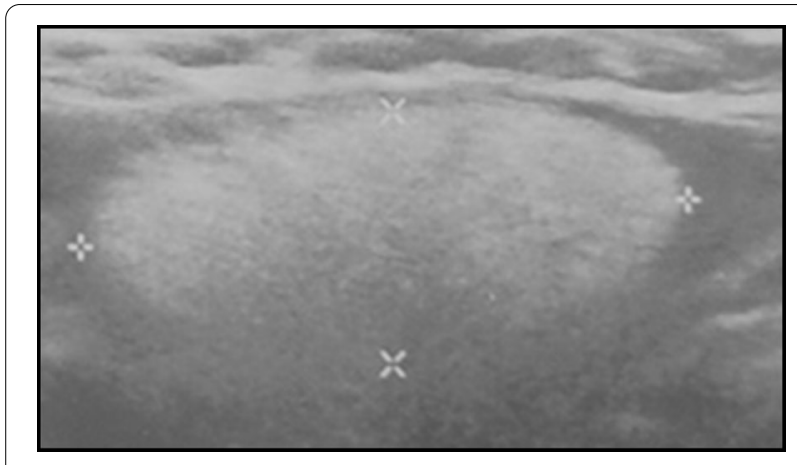

Fig. 2 Left hyper-echoic testicle

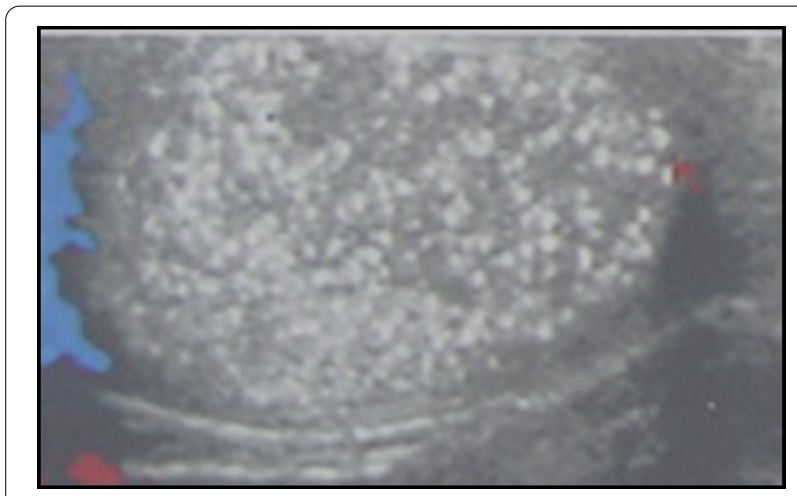

Fig. 3 Right testicle containing grade 3 TMs

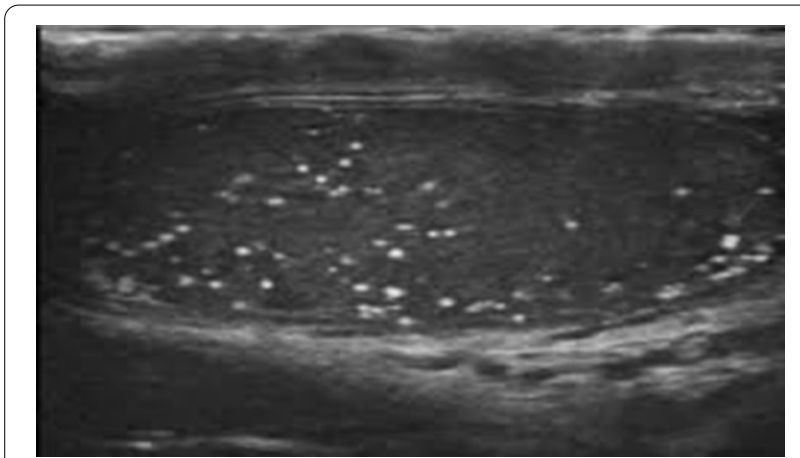

Fig. 4 Ultrasound image showing grade 3 TMs

irregularities in the testicular consistence in both sides, with the growth of TMs on the left side not detected before.

\subsection{Observation 3}

The 3rd child was 8 years old, with a medical history of hypothyroidism. He was referred for consultation for ectopic testis. The examination revealed that the right testicle was palpable, with a normal size and consistency. However, the testis inguinal position was abnormal, and the ultrasound images showed bilateral TMs (Fig. 4).

In this case, the child undertook an undescended testicle repair surgery and a biopsy on the right side. Different from the second case, this patient sample has not revealed any TMs in the biopsy fragment.

\subsection{Observation 4}

Our last patient was a 3-year-old child, previously diagnosed with right renal agenesis, referred for right ectopic testicular. Neither the physical examination nor the testicular ultrasound had detected any anomalies in the right testicle.

The exploratory laparoscopy allowed us to view the right testicle under liver atrophy with pedicle hypoplasia. The pathological examination of the orchiectomy sample revealed the presence of TMs.

\section{Discussion}

\subsection{The frequency of TMs}

A prospective study of asymptomatic pediatric population, of a frequency of $4.2 \%$, was performed [1]. Indeed, the children for whom the TMs diagnosis was to be carried out and having a scrotal ultrasound for targeted purposes (e.g., pain, trauma) fell between 1.1 and $8.6 \%[1,3]$. The significant disparity between these rates is explained by the pathology, which underlines the use of ultrasound depending on the age and ethnic groups, as well as races. The authors found a higher frequency of TMs between children from the white race and Asian population, with an age $<11$ years [1].

\subsection{The TMs pathogenesis}

There are many etiological theories of TMs that are still extensively debated. The majority of these theories converge to the hypothesis of multiple inter-relational phenomena. In fact, this phenomenon provokes a cell degeneration in addition to a Sertoli cells dysfunction, causing an inability to remove fragments. Hence, the TMs development is initiated $[1,2]$.

\subsection{The diagnosis methods}

The diagnosis of TMs is mainly performed by scrotal ultrasound for various reasons, either for benign testicular pathologies, cryptorchidism [4], testicular trauma, hydrocele, varicocele, and twisting annexes [5], or chromosomal abnormalities assessment, such as Klinefelter syndrome and Trisomy 21 [6]. 


\subsection{Clinical examination}

The clinical examination of children does not give evidence for the functional symptoms. Indeed, TMs are usually incidentally discovered in asymptomatic patients. Except for the cases published by Ducheket, a case of painful testicular microlithiasis suggested a distension of the seminiferous tubules [1].

The physical examination of these children validated unusual testicular consistency abnormality, affected by testicular atrophy, cryptorchidism, or as part of testicular pathology of a trauma or an infection.

\subsection{The explorations}

\subsubsection{Doppler testicular scrotal ultrasound}

The testicular ultrasound is an essential step for the positive diagnosis of TMs. In most cases, TMs can be bilateral and symmetrical, but also unilateral and asymmetrical. The ultrasound is also helpful in defining the TMs grade [7].

\subsection{Testicular biopsy}

According to some authors, testicular biopsy should be systematic and done as soon as the TMs are diagnosed through the ultrasound image. It would help to detect any tumor development at an early stage.

However, because of its invasive nature and its psychological impact, testicular biopsy is kept for particular cases: case of focal or unilateral TMs, ipsilateral TMs with germ cell tumors, or in case of suspicious changes in ultrasound images during the checkup. Still, cryptorchidism and testicular atrophy are considered as a debate subject [8].

\subsection{Laboratory tests}

The normality of calcium and phosphate dismisses the lithogenes which is theory of metabolic origin.

Furthermore, tumor markers are important for the majority of researchers, to be measured both during the first examination, and in subsequent follow-up when clinical or radiological changes are observed.

Besides, karyotype remains important for the search for chromosomal disorder associated with TMs.

\subsection{Pathological associations}

In addition to genetic diseases, karyotype is of significant interest in the treatment of this MT pathology, frequently associated with cryptorchidism and tumor.

\subsection{Cryptorchidism pathology}

Since cryptorchidism is usually associated with TMs pathology, it would be of interest to search for the link between the two entities.

The existence of TMs on undescended testes is explained by the sequela of a testicular atrophy with smaller diameter tubes, but also by the increase in cellular degradation on these painful testis $[1,9]$.

\subsection{Tumor pathology}

Although this link was explored in case of adults more than children, we will try this chapter to raise up many important questions, mainly:

1. Are TMs Lesions Predisposing to Testicular Cancer?

2. Could TMs Diagnosed During Childhood Develop To Cancer In Adulthood?

3. Are TMs Diagnosed In Children A Risk Factor For tumor Development During Childhood?

Before introducing the topic, it is important to point out that testicular cancers count only $1 \%$ of pediatric cancers, and 8 to $16 \%$ of this cancer have cryptorchidism history. Cryptorchidism increases the relative risk of cancer by $7.5 \%$. This risk is particularly important in cases of abdominal cryptorchidism or bilateral disease [10].

According to Furness, none of the 26 patients he followed for TMs during a median of 27.6 months had developed a tumor [11]. The same observation was made by Skyrme, who followed 5 patients for 45 months and concluded that none of them suffered from cancer [12]. In their study carried out in 2002, Leen and Riebel found 16 cases of TMs out of 850 scrotal ultrasonographies performed in symptomatic patients. However, none of them has developed a tumor [1]. For Dagasch and Machinnon's study done in 2007, 7 children were diagnosed with TMs out of 623 symptomatic patients based on ultrasound images. However, no case of tumor pathology was detected [1]. Similar to the previous authors, Kocaoglu and his colleagues had followed 9 children, aged from 6 months to 6 years, none of whom had developed any tumor [1]. In 2007, Miller et al. found 4 children out of 198 symptomatic ones with TMs, using scrotal ultrasonography. However, none of them have developed a neoplastic disease [1].

These authors supervised children with TMs, but none of those cases developed a tumor. Indeed, they could not link TMs to cancer, but this possibility cannot be disregarded since short follow-up does not allow for efficient detection of such pathology. 


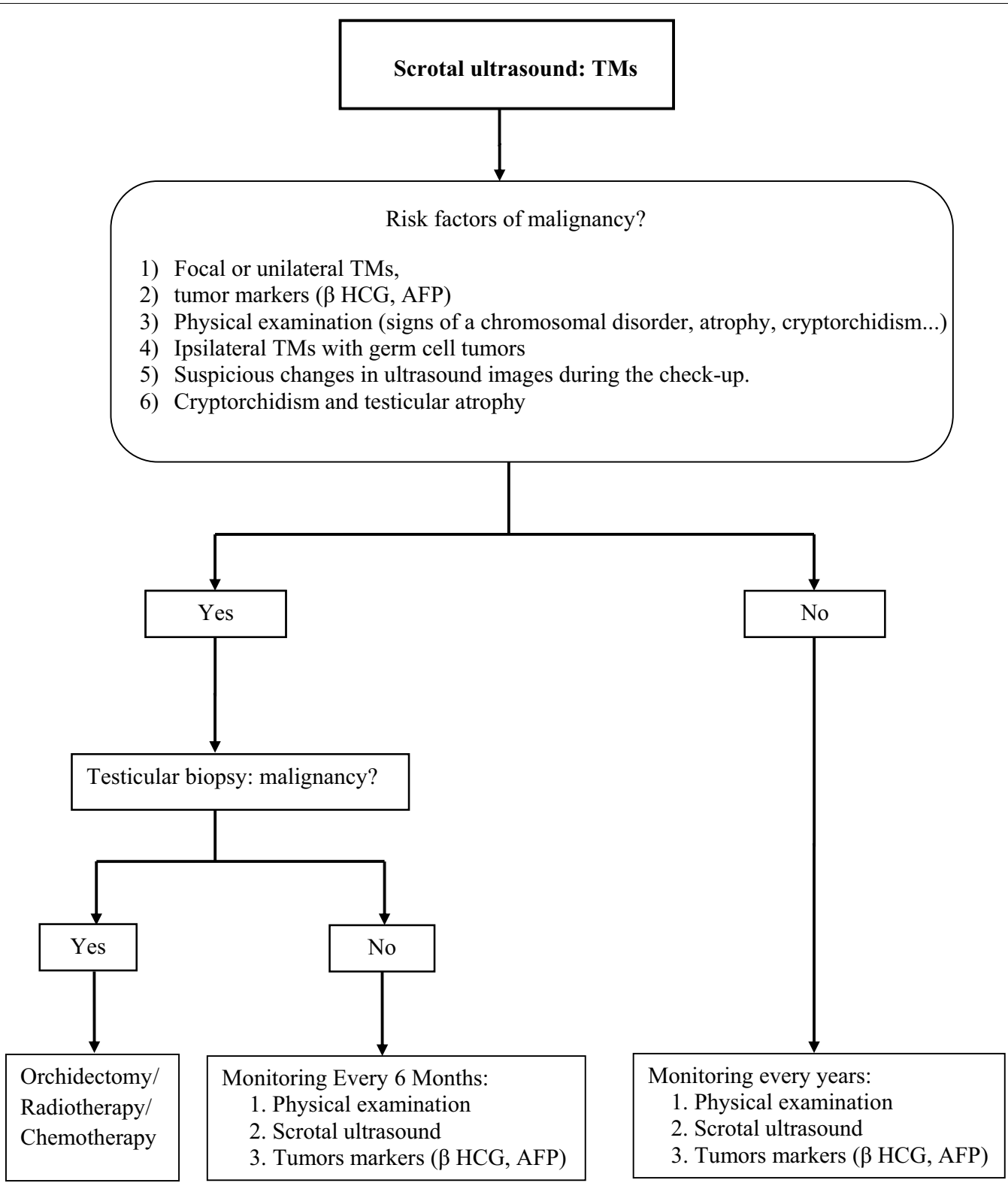

Fig. 5 Management algorithm of children diagnosed with TMs

In 1996, a case of 6-year-old child admitted for precocious pseudo-puberty was reported by Arrigot et al. During the examination, a hydrocele was diagnosed. Although the biological assessment was initially normal, the scrotal ultrasound and the testicular biopsy revealed TMs. Because of their large number, a biannual check-up was planned. However, the child did not undergo any follow-up, and as a result, he developed a yolk sac tumor at the age of 9. This tumor was discovered by another doctor whom he consulted for testicular asymmetry [1].
A case of an 11-year-old child was described by Bierger in 1965. At the age of 5 , the child was diagnosed with male pseudo-hermaphroditism and bilateral cryptorchidism with TM. Six years later, he developed a gonadoblastoma at the age of 11 [1].

In 1995, MC-Eniff et al. reported the case of a 17 -year-old adolescent admitted for an increase in testis volume, previously diagnosed with TMs. Vachan et al. did a study on 92 trisomy patients, with age ranging from 0 to 29 years. Twenty-one cases were already diagnosed with TMs, but only 1 case developed a 
benign tumor with Sertoli cells after 5 years of followup [13].

The most recent case was published in 2013 by Pica et al. describing the case of an 15-year-old adolescent, who developed testicular germ cell tumor ipsilateral to TMs unintentionally discovered through screening. A case of a child was described by Kang in 1994, which was diagnosed with a testicular tumor, in the presence of contralateral TMs with CIS [1].

According to our review and synthesis, these children developed germ cell tumors in childhood. These observations are very interesting, since they emphasize the existence of latency between the discovery of TMs and the development of a tumor, which is frequently too long.

\subsection{The management of children carrying TMs (Fig. 5)}

\subsubsection{Initial assessment}

- These children have to undergo a complete physical examination.

- Physicians have to seek in these histories: the notion of trauma, infection, associated tumor pathology, etc.

- A karyotype is necessary if the clinical examination reveals any signs of a chromosomal disorder.

- Doppler scrotal ultrasound and testicular biopsy are indicated in case of testicular atrophy or unilateral testicular germ or cell tumor with contralateral TMs. However, this overall examination is still to be discussed in the presence of unilateral TMs [14].

\subsection{Monitoring pace}

\subsubsection{In short term}

It is recommended to make a regular follow-up every year of children who present testicularmicrolithiasis anda closer follow-up at six months if there are any risque factors for Germinal Testicular Tumor (GTT) Fig. 5, through the following:

1. Physical examination

2. Scrotal ultrasound

3. Tumor markers

\subsubsection{In long term}

The transition between adolescence and adulthood is considered as a targeted age to develop testicular cancer.

\section{Conclusion}

There is a link between testicular microlithiasis and testicular cancer, and the transition between adolescence and adulthood is considered as a targeted age to develop testicular cancer. Therefore, it is recommended to make a regular follow-up of children who present testicular microlithiasis.

\section{Abbreviations}

TMs: Testicular microlithiasis; AFP: Alpha fetoprotein; CIS: Carcinoma in situ.

\section{Acknowledgements}

Not applicable.

\section{Authors' contributions}

* 'AC' collected, analyzed, and interpreted the patients' data and was the major contributor in writing the manuscript. * 'AFD' contributed in writing the manuscript. ' 'WH' and 'KK' checked the manuscript and made corrections. ' $R L$ ' and 'AN' helped in the final correction of the manuscript. All authors have read and approved the manuscript.

\section{Funding}

This research did not receive any specific grant from funding agencies in the public, commercial, or not-for-profit sectors.

\section{Availability of data and materials}

Not applicable.

\section{Declarations}

\section{Ethics approval and consent to participate}

The use of medical records was approved by the Ethics Committee at the Ministry of Health Hospital Fattouma Bourguiba of Monastir.

Consent for publication

Consent for publication of case descriptions was obtained from the parents of the patients.

\section{Competing interests}

The authors declare that they have no competing interests.

\section{Author details}

${ }^{1}$ Department of Urology, Fattouma Bourguiba Hopital, Monastir, Tunisia. ${ }^{2}$ Department of Pediatric Surgery, Fattouma Bourguiba Hopital, Monastir, Tunisia.

Received: 21 October 2020 Accepted: 11 July 2021

Published online: 21 August 2021

\section{References}

1. Goede J, Hack WWM, van der Voort-Doedens LM, Sijstermans K, Pierik $\mathrm{FH}$ (2009) Prevalence of testicular microlithiasis in asymptomatic males 0 to 19 years old. J Urol 182(4):1516-1520

2. Richie JP, Richie JP (2016) Testicular microlithiasis imaging and followup: guidelines of the ESUR Scrotal Imaging Subcommittee. J Urol 196(3):776

3. Chiang LW, Yap TL, Asiri MM, Ong CCP, Jacobsen AS (2012) Implications of incidental finding of testicular microlithiasis in paediatric patients. J Pediatric Urol 8(2):162-165

4. Yoko Nishimura, Kimihiko Moriya, Michiko Nakamura, Mutsumi Nishida, Nobuo Shinohara. Prevalence and Chronological Changes of Testicular Microlithiasis in Isolated Congenital Undescended Testes Operated at Less Than 3 Years of Age. Urology, Available online 2 August 2017 
5. Furness PD, Husmann DA, Brock JW, SteinhardT GF, Cheng EY (1998) Multi-institutional study of testicular microlithiasis in childhood: a benign or premalignant condition? J Urol 160(3):1151-1154

6. Goede J, Weijerman ME, Broers CJM, de Winter JP, Hack WWM (2012) Testicular volume and testicular microlithiasis in boys with down syndrome. J Urol 187(3):1012-1017

7. Canning DA (2016) Is ultrasound demonstration of testicular microlithiasis in pediatric patients: is there an association with testicular germ cell tumors? J Urol 196(1):226

8. Patel RP, Kolon TF, Huff DS, Carr MC, Snyder HM (2005) Testicular microlithiasis and antisperm antibodies following testicular biopsy in boys with cryptorchidism. J Urol 174(5):2008-2010

9. Goede J, Hack WWM, van der Voort-Doedens LM, Pierik FH, Sijstermans K (2010) Testicular microlithiasis in boys and young men with congenital or acquired undescended (ascending) testis. J Urol 183(4):1539-1544

10. Cos LR, Weinberg E, Messing EM, Rashid HH (2004) Testicular microlithiasis: a review and its association with testicular cancer. Urol Oncol Semin Origin Investig 22(4):285-289
11. Furness PD, Husmann DA, Brock JW, Earl GF, Cheng SY (1998) Multiinstitutional study of testicular microlithiasis in childhood: a benign or premalignant condition? J Urol 160(3):1151-1154

12. O'Malley PJ, Skyrme R, Jones AR, Fenn N, Bowsher WG (2003) Is testicular microlithiasis (TMs) a dangerous condition? Eur Urol Suppl 2(1):155

13. Vachon L, Fareau GE, Wilson MG, Chan LS (2006) Testicular microlithiasis in patients with down syndrome. J Pediatr 149(2):233-236

14. DeCastro BJ, Peterson AC, Costabile RA (2008) A 5-year followup study of asymptomatic men with testicular microlithiasis. J Urol 179(4):1420-1423

\section{Publisher's Note}

Springer Nature remains neutral with regard to jurisdictional claims in published maps and institutional affiliations.

\section{Submit your manuscript to a SpringerOpen ${ }^{\circ}$ journal and benefit from:}

- Convenient online submission

- Rigorous peer review

- Open access: articles freely available online

- High visibility within the field

- Retaining the copyright to your article

Submit your next manuscript at $>$ springeropen.com 